\title{
THE VERTEX-RAINBOW INDEX OF A GRAPH
}

\author{
YAPING $\mathrm{MAO}^{1}$ \\ Department of Mathematics \\ Qinghai Normal University \\ Qinghai 810008, China \\ e-mail: maoyaping@ymail.com
}

\begin{abstract}
The $k$-rainbow index $\operatorname{rx}_{k}(G)$ of a connected graph $G$ was introduced by Chartrand, Okamoto and Zhang in 2010. As a natural counterpart of the $k$ rainbow index, we introduce the concept of $k$-vertex-rainbow index $\operatorname{rvx}_{k}(G)$ in this paper. In this paper, sharp upper and lower bounds of $\operatorname{rvx}_{k}(G)$ are given for a connected graph $G$ of order $n$, that is, $0 \leq \operatorname{rvx}_{k}(G) \leq n-2$. We obtain Nordhaus-Gaddum results for 3-vertex-rainbow index of a graph $G$ of order $n$, and show that $\operatorname{rvx}_{3}(G)+\operatorname{rvx}_{3}(\bar{G})=4$ for $n=4$ and $2 \leq$ $\operatorname{rvx}_{3}(G)+\operatorname{rvx}_{3}(\bar{G}) \leq n-1$ for $n \geq 5$. Let $t(n, k, \ell)$ denote the minimal size of a connected graph $G$ of order $n$ with $\operatorname{rvx}_{k}(G) \leq \ell$, where $2 \leq \ell \leq n-2$ and $2 \leq k \leq n$. Upper and lower bounds on $t(n, k, \ell)$ are also obtained.
\end{abstract}

Keywords: vertex-coloring, connectivity, vertex-rainbow $S$-tree, vertexrainbow index, Nordhaus-Gaddum type.

2010 Mathematics Subject Classification: 05C05, 05C15, 05C40, $05 \mathrm{C} 76$.

\section{REFERENCES}

[1] M. Aouchiche and P. Hansen, A survey of Nordhaus-Gaddum type relations, Discrete Appl. Math. 161 (2013) 466-546.

doi:10.1016/j.dam.2011.12.018

[2] J.A. Bondy and U.S.R. Murty, Graph Theory (Graduate Texts in Mathematics 244, Springer-Verlag, London, 2008).

[3] Q. Cai, X. Li and J. Song, Solutions to conjectures on the $(k, \ell)$-rainbow index of complete graphs, Networks 62 (2013) 220-224. doi: $10.1002 /$ net.21513

\footnotetext{
${ }^{1}$ Supported by the National Science Foundation of China (Nos. 11551001 and 11161037) and the Science Found of Qinghai Province (No. 2014-ZJ-907).
} 
[4] Q. Cai, X. Li and J. Song, The $(k, \ell)$-rainbow index of random graphs, Bull. Malays. Math. Sci. Soc. 39 (2016) 765-771.

[5] Y. Caro, A. Lev, Y. Roditty, Z. Tuza and R. Yuster, On rainbow connection, Electron. J. Combin. 15 (2008) \#R57.

[6] G. Chartrand, G.L. Johns, K.A. McKeon and P. Zhang, Rainbow connection in graphs, Math. Bohem. 133 (2008) 85-98.

[7] G. Chartrand, G.L. Johns, K.A. McKeon and P. Zhang, The rainbow connectivity of a graph, Networks 54 (2009) 75-81.

doi: $10.1002 /$ net.20296

[8] G. Chartrand, O.R. Oellermann, S. Tian and H.B. Zou, Steiner distance in graphs, Časopis pro pěstování matematiky 114 (1989) 399-410.

[9] G. Chartrand, F. Okamoto and P. Zhang, Rainbow trees in graphs and generalized connectivity, Networks 55 (2010) 360-367. doi: $10.1002 /$ net.20340

[10] L. Chen, X. Li and H. Lian, Nordhaus-Gaddum-type theorem for rainbow connection number of graphs, Graphs Combin. 29 (2013) 1235-1247. doi:10.1007/s00373-012-1183-x

[11] L. Chen, X. Li and M. Liu, Nordhaus-Gaddum-type theorem for the rainbow vertex connection number of a graph, Util. Math. 86 (2011) 335-340.

[12] L. Chen, X. Li, K. Yang and Y. Zhao, The 3-rainbow index of a graph, Discuss. Math. Graph Theory 35 (2015) 81-94. doi: $10.7151 /$ dmgt.1780

[13] X. Cheng and D. Du, Steiner Trees in Industry (Kluwer Academic Publisher, Dordrecht, 2001).

[14] D. Du and X. Hu, Steiner Tree Problems in Computer Communication Networks (World Scientific, River Edge, 2008).

[15] M. Krivelevich and R. Yuster, The rainbow connection of a graph is (at most) reciprocal to its minimum degree, J. Graph Theory 63 (2010) 185-191. doi:10.1002/jgt.20418

[16] H. Li, X. Li, Y. Sun and Y. Zhao, Note on minimally d-rainbow connected graphs, Graphs Combin. 30 (2014) 949-955. doi:10.1007/s00373-013-1309-9

[17] X. Li, I. Schiermeyer, K. Yang and Y. Zhao, Graphs with 3-rainbow index $n-1$ and $n-2$, Discuss. Math. Graph Theory 35 (2015) 105-120. doi:10.7151/dmgt.1783

[18] X. Li, I. Schiermeyer, K. Yang and Y. Zhao, Graphs with 4-rainbow index 3 and $n-1$, Discuss. Math. Graph Theory 35 (2015) 387-398.

doi:10.7151/dmgt.1794 
[19] X. Li and Y. Shi, On the rainbow vertex-connection, Discuss. Math. Graph Theory 33 (2013) 307-313. doi:10.7151/dmgt.1664

[20] X. Li, Y. Shi and Y. Sun, Rainbow connections of graphs: A survey, Graphs Combin. 29 (2013) 1-38.

doi:10.1007/s00373-012-1243-2

[21] X. Li and Y. Sun, Rainbow Connections of Graphs (SpringerBriefs in Math., Springer, New York, 2012).

[22] Y. Mao and Y. Shi, The complexity of determining the vertex-rainbow index of graphs, Discrete Math. Algorithms Appl. 7(4) (2015) 1550047. doi:10.1142/s1793830915500470

[23] E.A. Nordhaus and J.W. Gaddum, On complementary graphs, Amer. Math. Monthly 63 (1956) 175-177.

[24] I. Schiermeyer, On minimally rainbow $k$-connected graphs, Discrete Appl. Math. 161 (2013) 702-705.

doi:10.1016/j.dam.2011.05.001

Received 11 September 2014

Revised 13 October 2015

Accepted 13 October 2015 